\title{
Epidermal Growth Factor Receptor Mutation Frequency in Squamous Cell Carcinoma and Its Diagnostic Performance in Cytological Samples: A Molecular and Immunohistochemical Study
}

\author{
Niraj Kumari ${ }^{a}$ e, Shalini Singh ${ }^{b}$, Dhanjit Haloia, Shravan Kumar Mishra, \\ Narendra Krishnani ${ }^{\mathrm{a}}$, Alok Nath ${ }^{\mathrm{c}}$, Zafar Neyaz ${ }^{\mathrm{d}}$
}

\begin{abstract}
Background: Epidermal growth factor receptor (EGFR) mutation is the most frequent mutation tested in lung cancer for targeted therapy in the era of personalized medicine. Knowledge about EGFR mutation is constantly expanding regarding its frequency, clinicopathological association, advancements in testing methodology and sample requirement. We investigated EGFR mutation frequency in non-small cell lung cancer (NSCLC) in North Indian patients and evaluated its diagnostic performance in cytological samples.
\end{abstract}

Methods: Molecular EGFR testing was done in 250 cases of NSCLC by both real-time polymerase chain reaction (PCR) (Therascreen) and mutation-specific EGFR immunohistochemistry (IHC). Thirty cases had both cytology samples and biopsy including 20 pleural effusions and 10 fine-needle aspirates. EGFR mutation concordance between pleural effusion and biopsy was studied.

Results: EGFR mutation was overall $31.6 \%$ in NSCLC with $36.5 \%$ in adenocarcinoma and $15 \%$ in squamous cell carcinoma. L858R mutation accounted for $50.7 \%$ and DEL19 for $39.3 \%$ of total EGFR mutations. Complex mutations were seen in $2 \%$ of cases. Sensitivity of mutation-specific EGFR IHC was $48.3 \%$ and specificity was $92.3 \%$. L858R showed higher sensitivity ( $55 \%$ vs. $33.3 \%$ ) but similar specificity $(93.2 \%$ vs. $91.3 \%)$ compared to DEL19. EGFR mutation was successful in $95 \%$ of pleural effusion and showed $83.3 \%$ concordance with tissue biopsy.

Manuscript submitted May 29, 2019, accepted June 10, 2019

aDepartment of Pathology, Sanjay Gandhi Postgraduate Institute of Medical Sciences, Lucknow 226014, India

bDepartment of Radiotherapy, Sanjay Gandhi Postgraduate Institute of Medical Sciences, Lucknow 226014, India

'Department of Pulmonary Medicine, Sanjay Gandhi Postgraduate Institute of Medical Sciences, Lucknow 226014, India

dDepartment of Radiodiagnosis, Sanjay Gandhi Postgraduate Institute of Medical Sciences, Lucknow 226014, India

${ }^{\mathrm{e}}$ Corresponding Author: Niraj Kumari, Department of Pathology, Sanjay Gandhi Postgraduate Institute of Medical Sciences, Lucknow 226014, India.

Email: nirajpath@gmail.com

doi: https://doi.org/10.14740/wjon1204
Conclusions: EGFR mutation frequency in North Indian patients was comparable to that of Asia-Pacific region and showed a similar pattern of histological distribution. EGFR mutation in squamous cell carcinomas is increasingly recognized which was $15 \%$ in our study. Mutation-specific EGFR IHC shows variable but generally low sensitivity and considering its significant pre- and post-analytical variables, it should be highly discouraged in patient management. Cytological samples may not only serve as suitable alternative but may be complementary to tissue biopsies.

Keywords: EGFR; Pleural effusion; Mutation-specific IHC; Squamous cell carcinoma; NSCLC

\section{Introduction}

Lung cancer is the most common cancer as well as the leading cause of cancer-related death. Over the years, understanding of lung cancer biology has evolved and the histological classification has been supplemented with molecular classification. Targetable driver mutations with development of oral tyrosine kinase inhibitors (TKIs) have changed the management of nonsmall cell lung cancer (NSCLC) dramatically. Of all the targetable mutations, epidermal growth factor receptor (EGFR) mutation is the most frequent and important marker in terms of management particularly in adenocarcinoma where TKIs have resulted in improved overall and progression-free survival with better tolerance compared to systemic chemotherapy.

The EGFR mutations have a wide prevalence all over the world being more prevalent in Asians [1-3]. Though EGFR mutations have been reported largely in lung adenocarcinoma, in the recent years, it is being increasingly reported in squamous cell carcinoma (SCC) as well in Asians, particularly in Chinese. Studies from Caucasians have reported very low rate of EGFR mutation in SCC $(0-3 \%)[4,5]$, while it is reported in the range of $0-19.2 \%$ in Chinese [3, 6, 7].

The early studies for EGFR mutation used mutationspecific EGFR immunohistochemistry (IHC) against two mutations (L858R, and DEL19) to detect EGFR mutations in NSCLC. These two mutations account for $80-90 \%$ of to- 
tal EGFR mutations. However, IHC results have shown lower sensitivity with both false-positive and false-negative results [8-10]. Intra-tumoral heterogeneity in IHC staining as well as pre-analytical and analytical variability is the major problems with EGFR mutation-specific antibodies. According to the current recommendations molecular methods such as quantitative real-time polymerase chain reaction (qPCR) is the method of choice for detecting EGFR mutations; however mutation-specific IHC is advised only for small biopsies having scant tumor cells where molecular testing is not feasible. In resource poor centers, mutation-specific EGFR IHC is still a viable option for guiding patient management [11].

One of the other issues relevant to EGFR mutation is that of sample type. Since large number of cases are detected in late stages particularly with effusion or poor patient performance scores, accessing tissue biopsy or even fine-needle aspirate cytology (FNAC) for EGFR testing becomes difficult. Studies are being done on malignant pleural effusion and peripheral blood samples to detect targetable mutations in NSCLC. Comparison studies between malignant pleural effusion and biopsy samples have shown good concordance between the two suggesting effusion samples to be an alternative of tissue biopsy in advanced stage NSCLC patients [12-15].

We studied the concordance of EGFR mutation by qPCR and mutation-specific EGFR IHC, as well as its feasibility in malignant effusion samples.

\section{Materials and Methods}

All patients with NSCLC after histological/cytological diagnosis, from November 2015 to April 2019, were tested for EGFR. Histological or cytological diagnosis was reviewed and IHC for TTF1, P63 and CK5/6 was used wherever necessary.

Paraffin blocks of endobronchial biopsies, cell blocks of fine-needle aspirates (FNAs) or effusion fluids were used to extract DNA using QIAamp extraction kit (Qiagen, Hilden, Germany). EGFR testing was done by qPCR using Qiagen Therascreen kiton QuantStudio 6 Flex real-time thermal cycler (Life Technologies, Carlsbad, CA, USA) according to the recommended protocol. Briefly, in the first step, samples were tested with internal control provided with the kit. Once the amplification was successful, then in second step primer, probes for 29 different mutations were tested in eight wells for each patient. $\Delta \mathrm{Ct}$ method was used to detect EGFR mutation according to the recommended cut-offs provided.

Mutation-specific IHC was used for exon 19 deletion (E746-A750) and L858R in exon 21 from Cell Signalling Technologies (Danvers, MA, USA) (dilution, 1:50; clone, 6B6 and 43B2 respectively). Membranous staining of $2+$ (strong incomplete membranous) or $3+$ (strong complete membranous) was considered as positive staining.

The EGFR mutation concordance between biopsy and effusion samples and that between qPCR and mutation-specific IHC were evaluated. Categorical variables were analyzed by Chi-square test and survival was analyzed by Kaplan Meier log rank test using SPSS version 20.

The study was approved from the Institute's Ethics Com- mittee vide letter no. IEC-2016-01-IMP-89. It was conducted in compliance with the ethical standards of the responsible institution on human subjects as well as with the Helsinki Declaration and informed consent was obtained from the patients.

\section{Results}

Five hundred thirty patients were diagnosed as NSCLC (adenocarcinoma, SCC, NSCLC-not otherwise specified (NOS)), of whom 250 patients were tested for EGFR mutation. EGFR testing was done based on tissue adequacy, patient's willingness for molecular testing and receiving anti-EGFR therapy. There were 175 men and 75 women with an age range of 26 87 years (mean: 59.3 years, median: 60 years). Pleural effusion was present in 99 patients of whom $45(45.5 \%)$ had malignant effusion. Biopsies alone were available in 205 patients, FNAC alone in eight patients and malignant effusion alone was available in 16 patients. In another 21 patients, both biopsy and malignant effusion (paired samples) were available. Mutationspecific IHC was done in 112 patients.

Of the 226 biopsies including 21 paired samples, adenocarcinoma comprised of 169 cases $(74.8 \%)$ followed by 43 cases of SCC (19.5\%), 10 cases of NSCLC-NOS (4.4\%) and three cases of adenosquamous (1.3\%). Thirty-six of the above cases were NSCLC-NOS of which 22 cases turned out to be adenocarcinoma, and four as SCC. Rest remained as NSCLC. Additionally only cytological material was present in 24 cases of which 16 were pleural effusion and eight were transbronchial FNAs. This included $20(83.3 \%)$ adenocarcinoma, three $(12.5 \%)$ SCC and one (4.2\%) NSCLC-NOS. So finally including both histology and cytology samples, there were 189 cases of adenocarcinoma, 46 cases of SCC and 11 cases of NSCLC.

\section{EGFR mutation}

EGFR mutation was found in $31.6 \%(79 / 250)$ of all patients with NSCLC. The two most common mutations, L858R (40 cases, 50.7\%) and DEL19 (31 cases, 39.3\%) together accounted for $90 \%$ of all mutations. Other mutations comprised of G719X (four cases, 5\%), T790M (two cases, 2.5\%) and INS (two cases, 2.5\%). Five cases harbored double EGFR mutations. Of these four were adenocarcinoma which showed L8585R + INS in two cases, L858R + DEL19 in one case and DEL19 + G719X in one case. The fifth case was that of SCC harboring L858R + T790M mutation.

EGFR mutation was higher in women $(40.5 \%)$ than in men $(28.3 \%)(\mathrm{P}$ value $=0.05)$ and in young patients with age $\leq$ 40 years $(47 \%$ vs. $30.7 \%$, P value $=0.1)$. Patients with pleural effusion $(38.8 \%$ vs. $27.5 \%$, $\mathrm{P}$ value $=0.06)$ or malignant pleural effusion $(43.2 \%$ vs. $18.2 \%$, P value $=0.1)$ also had higher EGFR mutation rate. Smoking was present in 115 patients of whom $36.8 \%$ were mutant whereas $63.2 \%$ of non-smokers showed EGFR mutation $(\mathrm{P}$ value $=0.05)($ Table 1$)$.

Adenocarcinoma showed 36.5\% (69/189) EGFR mutation followed by $15 \%(7 / 46)$ in SCC, $27.3 \%(3 / 11)$ in NSCLC and none $(0 / 3)$ of the three cases of adenosquamous carcinoma $(\mathrm{P}$ 
Table 1. EGFR Genotype With Clinicopathological Parameters

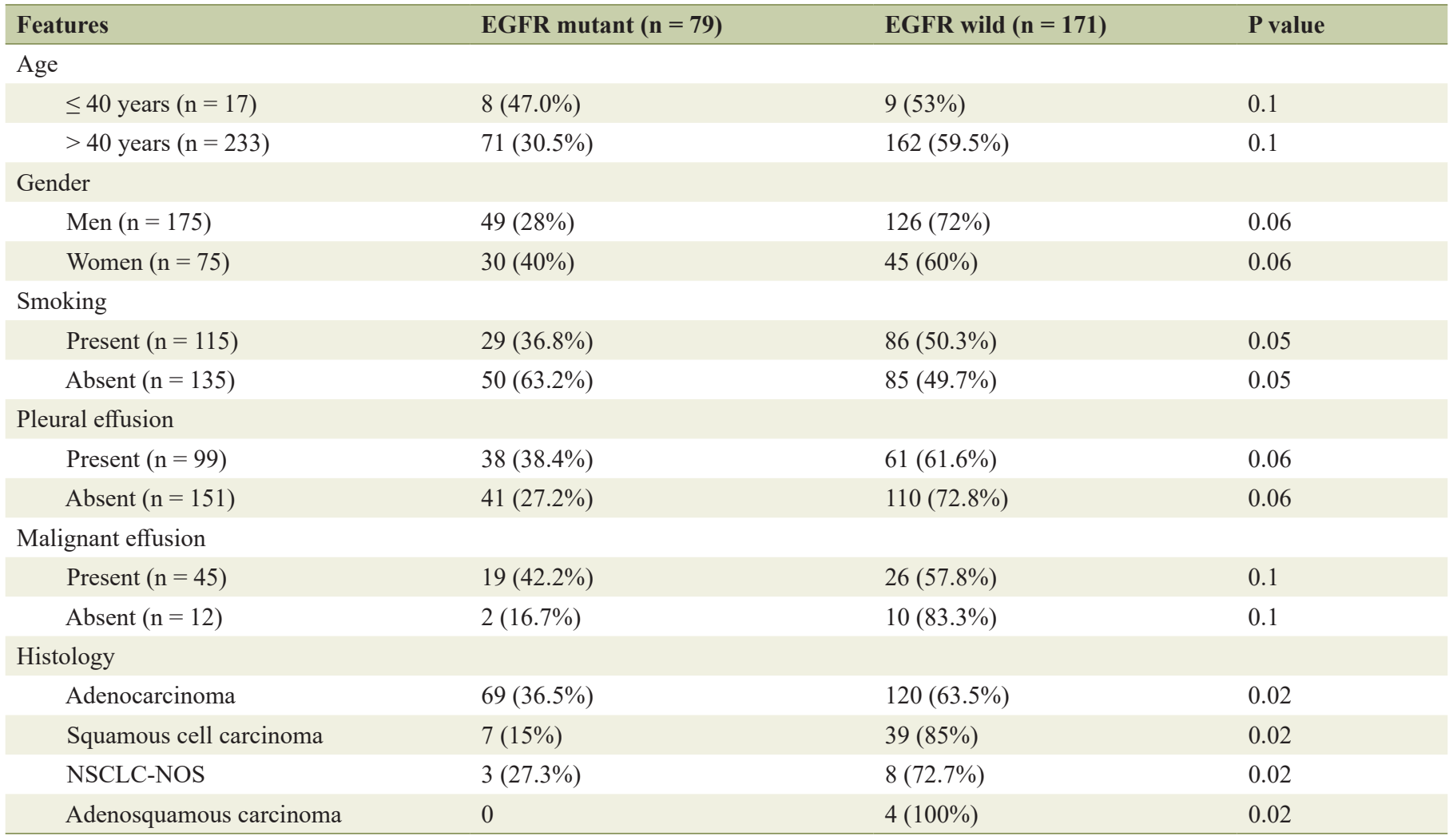

value $=0.02)$. Similar to adenocarcinoma, L858R was the frequent mutation in SCC followed by DEL19, G719X and INS. Thus, EGFR mutation pattern in both the histological types was largely similar.

\section{Mutation-specific EGFR IHC}

Mutation-specific EGFR IHC was done in 112 cases for L858R and DEL19 of which 29 cases were mutant for L858R (20 cases) and DEL19 (nine cases) on qPCR whereas mutantspecific IHC was positive for L858R in 19 cases and DEL19 in 10 cases. Mutation-specific IHC correctly picked up 11/20
(55\%) cases of L858R and 3/9 (33.3\%) cases of DEL19 (Table 2 ). The sensitivity and specificity of mutation-specific EGFR antibodies were calculated considering the molecular testing as gold standard. Both the antibodies together showed a sensitivity of $48.3 \%$ and specificity of $92.3 \%$ with L858R IHC having greater sensitivity (55\% vs. $33.3 \%)$ but nearly similar specificity (93.2\% vs. 91.3\%) than DEL19 IHC (Table 3).

\section{EGFR testing in cytological specimens}

EGFR was tested in 30 cases having paired biopsy and cytology samples (pleural effusion: 20 cases, FNA: 10 cases). Of the

Table 2. Comparison Between EGFR Mutation by Real-Time PCR and Immunohistochemistry

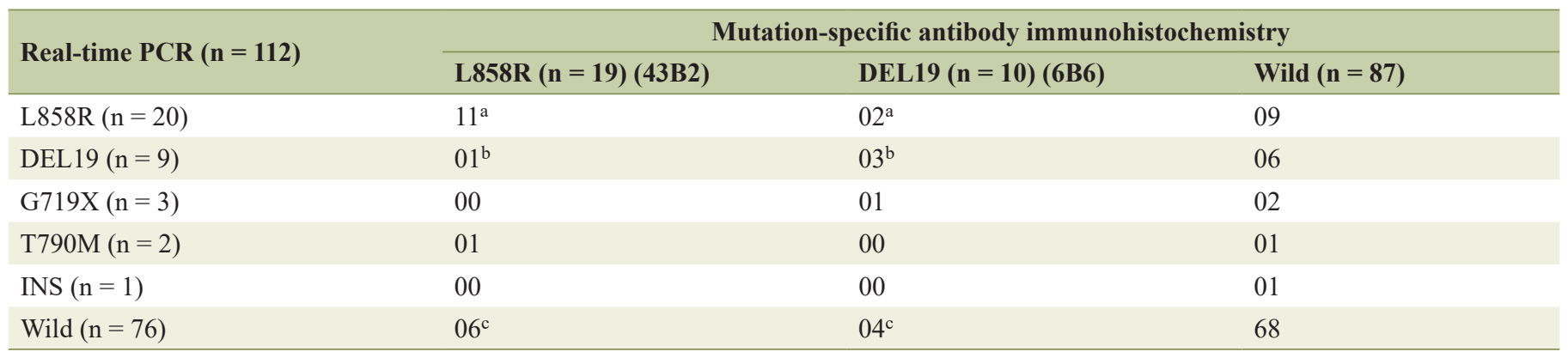

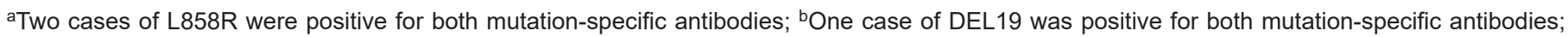
'One case of wild type was positive for both mutation-specific antibodies. 
Table 3. Sensitivity and Specificity of Mutation-Specific EGFR Antibodies

\begin{tabular}{lllllll}
\hline Mutation-specific antibodies & True positive & True negative & False positive & False negative & Sensitivity (\%) & Specificity (\%) \\
\hline L858R (n=112) & 11 & 84 & 08 & 09 & 55 & 91.3 \\
DEL $(\mathrm{n}=112)$ & 03 & 96 & 07 & 06 & 33.3 & 93.2 \\
Total & 14 & 180 & 15 & 15 & 48.3 & 92.3 \\
\hline
\end{tabular}

10 cases having FNA and biopsy, EGFR mutation was detected in 2/10 cases in biopsy (L858R: one case, DEL19: one case) and 4/10 cases in FNA with two additional cases (L858R: two cases, DEL19: two cases). In 20 cases with pleural effusion and biopsy, one case had very scant malignant cells and did not amplify; however it was successfully amplified in biopsy sample. Another case that did not show amplification on biopsy was successful on effusion sample. Thus, mutation testing in effusion samples was successful in 95\% (19/20) cases. The concordance rate of EGFR mutation between biopsy and effusion (18 paired cases) was $83.3 \%(15 / 18)$ as effusion samples showed mutation in three extra cases (16.7\%) (3/18) (L858R: one, G719A: two), where biopsy was wild, giving a higher mutation detection rate in effusion sample $(64.7 \%)$ as compared to biopsy (47\%) (Table 4). Thus considering all the cytological samples together, EGFR mutation was detected in $10 / 30(33.3 \%)$ biopsy samples and $15 / 30(50 \%)$ of cytological samples.

Follow-up was available in 228 cases ranging from 2 53 months with a mean follow-up duration of 7.6 months and median of 4 months. Fifteen deaths were recorded. In EGFR wild patients mean and median survival was 6.8 and 4 months whereas in EGFR mutant it was marginally higher with 9.4 and 5.5 months $(\mathrm{P}$ value $=0.5)$ respectively.

\section{Discussion}

Lung cancer bears the load of cancer worldwide with approximately $11.6 \%$ incidence and $18.4 \%$ cancer-related mortality. In India, nearly 67,800 new cases and 48,700 deaths related to lung cancer were reported in 2018 according to the GLOBOCAN 2018 India factsheet [16]. The role of EGFR TKIs in patients with NSCLC harboring EGFR mutations has emerged as a key oncogenic outcome and EGFR status has become a major prognostic factor in lung cancer. A study by Midha et al as well as those by others $[2,8,17-23]$ have shown EGFR mu- tation frequency to be highest in Asia-Pacific region averaging $47 \%$ but with a very wide range of $20-76 \%$ in different parts of this region. They also showed that the wider variability of EGFR mutation status was prevalent within each country of the specific regions of world.

India is a culturally and ethnically versatile country. In the present study from northern part of India which caters patients largely from the state of Uttar Pradesh and its neighboring states such as Bihar, Orissa and country like Nepal showed an overall EGFR mutation frequency of $31.6 \%$ in NSCLC, irrespective of histology, which is similar to the previously published EGFR mutation frequency reported from Asia-Pacific region. Other studies from India which have been mostly reported from south and central parts of India had shown EGFR mutation ranging between 16-43\% [24-26] with two studies from North India itself reported by Maturu et al [25] and Kasana et al [26] showed EGFR mutation frequency of $16.6 \%$ and $35.1 \%$ respectively. This shows that the genetic changes are influenced by various environmental factors, geographical variations, ethnicity and lifestyle habits. From the Asia-Pacific region, Taiwan shows highest EGFR mutation of 57\% (range 36-76\%), while Singapore shows the lowest frequency of $40 \%$ (range $39-43 \%$ ). However, in the extreme south-east Australia lowest EGFR mutation frequency ranging between $7-36 \%[27,28]$ was reported. The widest range of EGFR mutation in NSCLC has been reported from South America ranging between 9-67\% with an overall frequency of $36 \%[2,29,30]$. Reports from Europe also show great variability with mutation frequency ranging between 7.3$40.8 \%$ (Table 5) [4, 8, 17-27, 29-40].

The combined mutation frequency of DEL19 and L858R was $89.8 \%$ similar to the published literature; however we found L858R to be higher than DEL19 with 50.6\% and 39.2\% respectively. Similar frequency of higher L858R mutation than DEL19 has been reported by Kim et al $(53.3 \%, 40.3 \%)$ and Yotsukura et al $(56.3 \%, 40 \%)[8,17]$. Complex mutations with more than one type of EGFR mutations were found in $5 / 250$

Table 4. Comparison of EGFR Mutation Between Biopsy and Malignant Effusion Specimen

\begin{tabular}{llllllll}
\multirow{2}{*}{ Biopsy specimen $(\mathbf{n}=\mathbf{2 0})$} & \multicolumn{7}{c}{ Malignant effusion specimen $(\mathbf{n}=\mathbf{2 0})$} \\
\cline { 2 - 7 } & $\mathbf{L 8 5 8 R}$ & DEL19 & T790M & G719A & INS & Wild & Unsuccessful \\
\hline L858R & 5 & - & - & - & - & - & 1 \\
DEL19 & - & 3 & - & - & - & - & - \\
T790M & - & - & - & - & - & - & - \\
G719A & - & - & - & - & - & - & - \\
INS & - & - & - & - & - & - & - \\
Wild & 1 & - & - & 2 & - & 7 & - \\
Unsuccessful & - & - & - & - & - & 1 & - \\
\hline
\end{tabular}


Table 5. Frequency Distribution of EGFR Mutation in Non-Small Cell Lung Cancer

\begin{tabular}{|c|c|c|c|c|}
\hline Area & Authors & Country & Year & EGFR mutation (\%) \\
\hline \multirow[t]{8}{*}{ Europe } & Leduc et al [32] & France & 2017 & 37 \\
\hline & Isaksson et al [27] & Sweeden & 2013 & 11 \\
\hline & De Greve et al [34] & Belgium & 2016 & 27 \\
\hline & Gervas et al [31] & Serbia & 2015 & 19 \\
\hline & Schmid et al [36] & Austria & 2009 & 7.3 \\
\hline & Arrieta et al [37] & Mexico & 2015 & 27.0 \\
\hline & Martin et al [38] & Canada & 2016 & 29.7 \\
\hline & Lopez-Chavez et al [39] & USA & 2016 & 21.9 \\
\hline \multirow{6}{*}{ Asia-Pacific } & Yotsukura et al [17] & Japan & 2017 & 46.9 \\
\hline & Rahman et al [18] & Japan & 2014 & 22.9 \\
\hline & Kim et al [8] & Korea & 2015 & 40.3 \\
\hline & Toh et al [20] & Singapore & 2010 & 39 \\
\hline & Chang et al [21] & Taiwan & 2007 & 76.5 \\
\hline & Shi et al [22] & Vietnam & 2014 & 64 \\
\hline \multirow[t]{2}{*}{ Australia } & Cooper et al [23] & Australia & 2013 & 14.7 \\
\hline & Sriram et al [40] & Australia & 2011 & 7.1 \\
\hline India & Kasana et al [26] & India & 2016 & 35.1 \\
\hline
\end{tabular}

( $2 \%$ ) cases. Complex EGFR mutations have also been reported in the similar range (2-3.4\%) in other studies [28, 41-43].

EGFR mutation frequency in NSCLC irrespective of any region has shown a consistent association with female gender. In the present study, $40 \%$ women and $28 \%$ men showed EGFR mutation which is similar to other studies like $28 \%$ versus $19 \%$ in North America, 22\% versus 9\% in Europe, 60\% versus 37\% in Asia-Pacific region and $48 \%$ versus $8 \%$ in Africa in women and men respectively [2]. Liu et al from China [13] (54.95\% men, $71.6 \%$ women) and Hsu et al from British Columbia (19\% men, $24.5 \%$ women) also reported similar female predilection for EGFR mutation [43]. Other studies from India have also shown similar female predominance in EGFR mutation $[1,17]$. A study from Bangladesh reported an exception to this pattern where Rahman et al reported higher EGFR mutation rate in men than women ( $25.5 \%$ men, $14.3 \%$ women $)$ [18].

\section{Histology versus mutation}

EGFR mutation has been largely associated with adenocarcinoma; however, in the last few years EGFR mutation is con- sistently being reported in SCC, both in endobronchial biopsies where it may be considered as component of adenosquamous carcinoma and in pure resected specimens with squamous histology proven by IHC. In the present study EGFR mutation was $36.5 \%$ in adenocarcinoma and $15 \%$ in SCC. L858R was also more frequent $(6.4 \%)$ in SCC followed by DEL19 (4.6\%), G719X (2\%) and INS (2\%). Studies from China and Korea have reported EGFR mutation in SCC ranging from 2-19\% [6, 7] whereas reports from American and European cohorts have shown lower frequency of SCC ranging from $0-2 \%[5,44,45]$. Han et al reported 9.9\% and 3.7\% EGFR mutation in SCC and $49.3 \%$ and $18 \%$ mutation in adenocarcinoma from Asia-Pacific region and Russia respectively [3]. EGFR mutation in SCC varies between $0-25 \%$ in different regions of the world $[6,29,31]$.

\section{EGFR mutation by mutation-specific antibodies}

EGFR mutation detection by using mutation-specific IHC for exon 19 deletion (E746-750) and exon 21 (L858R) point mutation are recommended for very small biopsies which have insufficient tumor cells for molecular testing. However, it is still 
Table 6. Sensitivity and Specificity of Mutation-Specific EGFR IHC With Molecular Testing

\begin{tabular}{lllll}
\hline Author & Country & Year & Sensitivity (DEL19 + L858R) & Specificity (DEL19 + L858R) \\
\hline Brevet et al [9] & USA & 2010 & 84 & 98.9 \\
Kitamura et al [47] & Japan & 2010 & 47 & 96 \\
Seo et al [10] & Korea & 2014 & 76.6 & 89 \\
Kim et al [8] & Korea & 2015 & 75.6 & 94.5 \\
Zhang et al [46] & China & 2016 & 83.7 & 98.6 \\
\hline
\end{tabular}

practiced in low resource centers due to unavailability of infrastructure and expertise. The sensitivity and specificity of EGFR mutation vary widely between different studies (sensitivity 47 $84 \%$, specificity $89-99 \%$ ), which may be due to the inherent technical problems of IHC [8-10, 40, 46-47]. In the present study EGFR mutation-specific IHC was available in 112 cases, of which 19 cases showed L858R mutation with a sensitivity and specificity of $55 \%$ and $91.2 \%$ respectively. DEL19 was detected in 10 cases by IHC with a sensitivity and specificity of $33.3 \%$ and $93.1 \%$ respectively. The overall specificities for the two mutation-specific antibodies have been reported to be high $(77-100 \%)$; however the sensitivity has been quite low (47-87.7\%). Sensitivity for E746-A750 deletion on IHC is 40$100 \%$ and for L858R point mutation is $36-100 \%$ [8-10]. The E746-A750 specific antibody detects the common 15 base pair deletion, which accounts for only $66-81 \%$ of E746-A750 deletion missing out approximately $20-34 \%$ of the exon 19 deletion $[8,9,40,47]$. Other reasons for low expression may be the different cut-offs of IHC pattern and intensity of expression considered used in different studies. Most studies have included incomplete $(2+)$ or complete $(3+)$ membranous staining as positive whereas a few others have included all grades of staining $(1+, 2+$ and $3+$ pattern $)$ as positive. The interobserver variability in interpretation of IHC is also one of the important reasons of variable sensitivity and specificity. Ragazzi et al showed the sensitivity and specificity for DEL19 (E746-750) IHC as 56\% and $100 \%$, respectively and sensitivity and specificity for exon 21 (L858R point mutation) as $70.4 \%$ and $89 \%$, respectively [45]. Zhang et al from China included all grades of staining as positive (1+ to $3+$ staining) and showed a sensitivity and specificity of $99.6 \%$ and $99.3 \%$, respectively for L858R whereas low sensitivity ( $86.0 \%$ and $82.7 \%$ respectively) for DEL19 (Table 6) $[8-10,46,47]$. The overall sensitivity and specificity of mutation-specific EGFR IHC were $48.3 \%$ and $92.2 \%$ in the present study. Our IHC results of DEL19 (E746-750) gave more inconsistent results than for L858R. DEL19 IHC showed more commonly cytoplasmic staining most of the time with either weak or moderate intensity and was not crisp giving a blurred transition from positive to negative stained field whereas in L858R, the staining was crisper and more intense with well delineated difference between positive- and negative-stained area.

\section{EGFR mutation in cytology samples}

Mutation detection in effusions has several potential advantages over analysis of tissue biopsies. First, tumor samples from patients with lung cancer are often limited, particularly when obtained from bronchial washings or fine-needle aspiration. These samples are used mainly for pathological diagnosis, frequently leaving insufficient numbers of cancer cells to be tested for an increasing number of targetable genomic abnormalities in addition to EGFR mutations, like ALK and ROS1. Because of the risks of complications from procedures used to obtain a tissue diagnosis and the frequent requirement for prompt initiation of therapy in patients with metastatic lung disease, some patients with targetable mutations may not be exposed to the effective drugs. The current standard for detection of EGFR mutations is tissue biopsy; however, obtaining tissue is one of the challenges in management of lung cancer. Cytological specimen particularly pleural effusion or a metastatic neck lymph node is valuable minimally invasive sample for EGFR mutation testing in advanced stage NSCLC patient. Though there is smaller chance of DNA degradation in cytology samples, the poor cellularity remains a major concern for false-negative tests particularly in effusion samples.

EGFR mutation was detected in 10/30 (34.5\%) biopsy samples and 15/30 (51.7\%) of cytological samples in the present study. The EGFR mutation was successfully detected in $95 \%$ of pleural effusion. In both paired biopsy and FNA samples, mutation detection rate was 20\% (2/10) and 40\% (4/10) respectively, while in the paired biopsy and pleural effusion it was $61.1 \%(11 / 18)$ and $44.4 \%(8 / 18)$ respectively. Three cases (16.7\%) showed wild EGFR genotype on biopsy but a mutant genotype effusion with L858R in one and G719A mutations in other two cases. This discrepancy could be either because of heterogeneity of tumor cells or presence of very scant mutant cells in the biopsy $(<5 \%)$ that were not picked up by qPCR. Liu et al showed a sensitivity of $81.8 \%$ and specificity of $80 \%$ for EGFR mutation detection in effusion sample with tissue biopsy sample [12]. Davies et al had successful EGFR mutation analysis in nine of $10(90 \%)$ cases with malignant pleural effusion [14]. Liu et al studied 192 paired malignant effusion and biopsy and found a higher mutation rate in biopsy of $62 \%$ compared to effusion of $58.9 \%$. They found concordance between biopsy and effusion in $87 \%$ cases similar to the present study [13]. Guan et al also showed higher mutation rate on biopsies of $34 \%$ compared to $30 \%$ on effusions with a concordance of $88.2 \%$ [15]. Although the mutation rate in different studies was somewhat higher in biopsies than in effusions, the present study showed opposite results; however no statistical difference was noted in any of the studies. Malignant effusion samples are easier to obtain in patients with advanced stage of disease as minimally invasive technique. The DNA quality also remains 
superior to biopsy which has the disadvantage of formalin fixation. Thus malignant effusions may be considered complementary to biopsies in management of NSCLC patients.

\section{Conclusions}

The present study shows EGFR mutation from a tertiary care referral academic institution in northern part of India with $36.5 \%$ in adenocarcinoma and $31.6 \%$ in overall NSCLC similar to that reported from Asia-Pacific region. Female gender predilection for EGFR mutation is also similar to the worldwide data. Our observations were different from the reported studies regarding frequencies of DEL19 and L858Rmutations. We found L858R mutation higher than DEL19 which has been reported in few studies from Japan, China and Korea. EGFR mutation in SCC has been largely reported from Asia-Pacific region. The present study also showed $15 \%$ EGFR mutation in $\mathrm{SCC}$ on the similar trend. Although role of anti-EGFR therapy in SCC is still in its naive phase, there are fair evidences for trials in this histological group. The current international guidelines do not recommend EGFR mutation-specific IHC except for cases where tumor tissue is insufficient for molecular studies, but still it is practiced in centers with limited resources. Considering the variable but low sensitivity of mutation-specific IHC with a lot of analytical variables involved, it is not suitable for routine use. Cytological samples in any form such as effusion fluids or FNAs are good alternatives to tissue biopsies in cases where biopsies are not accessible. In the present study, cytological samples yielded a better mutation yield than biopsy which may be due to better preservation of DNA as well as absence of contaminating non-tumorous cells and supporting tissues which could be there in biopsies. Cytological samples should be used as complementary to tissue biopsy rather than as alternative where both are available because there is always a chance of tumor heterogeneity in these tumors.

\section{Acknowledgments}

We would like to thank our patients, Department of Radiotherapy and the Institute's Research Committee.

\section{Financial Disclosure}

The work was funded by the Institute's intramural research funding.

\section{Conflict of Interest}

None.

\section{Informed Consent}

Informed consent was obtained from the patients.

\section{Author Contributions}

N Kumari, N Krishnani, SS, AN and ZN contributed to conception and design of the study; N Kumari, DH, SKM, SS, AN and $\mathrm{ZN}$ contributed to acquisition of data; $\mathrm{N}$ Kumari, DH, and SKM were involved in analysis and interpretation of data; $\mathrm{N}$ Kumari, DH, and N Krishnani contributed to drafting and revising the article; N Kumari, N Krishnani gave final approval of the version to be submitted.

\section{References}

1. Chougule A, Prabhash K, Noronha V, Joshi A, Thavamani A, Chandrani P, Upadhyay P, et al. Frequency of EGFR mutations in 907 lung adenocarcioma patients of Indian ethnicity. PLoS One. 2013;8(10):e76164.

2. Midha A, Dearden S, McCormack R. EGFR mutation incidence in non-small-cell lung cancer of adenocarcinoma histology: a systematic review and global map by ethnicity (mutMapII). Am J Cancer Res. 2015;5(9):2892-2911.

3. Han B, Tjulandin S, Hagiwara K, Normanno N, Wulandari L, Laktionov K, Hudoyo A, et al. EGFR mutation prevalence in Asia-Pacific and Russian patients with advanced NSCLC of adenocarcinoma and non-adenocarcinoma histology: The IGNITE study. Lung Cancer. 2017;113:37-44.

4. Chatziandreou I, Tsioli P, Sakellariou S, Mourkioti I, Giannopoulou I, Levidou G, Korkolopoulou P, et al. Comprehensive molecular analysis of NSCLC; Clinicopathological Associations. PLoS One. 2015;10(7):e0133859.

5. Skov BG, Hogdall E, Clementsen P, Krasnik M, Larsen KR, Sorensen JB, Skov T, et al. The prevalence of EGFR mutations in non-small cell lung cancer in an unselected Caucasian population. APMIS. 2015;123(2):108-115.

6. Zhang Q, Zhu L, Zhang J. Epidermal growth factor receptor gene mutation status in pure squamous-cell lung cancer in Chinese patients. BMC Cancer. 2015;15:88.

7. Lee HY, Lee SH, Won JK, Lee DS, Kwon NJ, Choi SM, Lee J, et al. Analysis of fifty hotspot mutations of lung squamous cell carcinoma in never-smokers. J Korean Med Sci. 2017;32(3):415-420.

8. Kim CH, Kim SH, Park SY, Yoo J, Kim SK, Kim HK. Identification of EGFR Mutations by Immunohistochemistry with EGFR Mutation-Specific Antibodies in Biopsy and Resection Specimens from Pulmonary Adenocarcinoma. Cancer Res Treat. 2015;47(4):653-660.

9. Brevet M, Arcila M, Ladanyi M. Assessment of EGFR mutation status in lung adenocarcinoma by immunohistochemistry using antibodies specific to the two major forms of mutant EGFR. J Mol Diagn. 2010;12(2):169176.

10. Seo AN, Park TI, Jin Y, Sun PL, Kim H, Chang H, Chung JH. Novel EGFR mutation-specific antibodies for lung adenocarcinoma: highly specific but not sensitive detection of an E746_A750 deletion in exon 19 and an L858R mutation in exon 21 by immunohistochemistry. Lung Cancer. 2014;83(3):316-323. 
11. Lindeman NI, Cagle PT, Beasley MB, Chitale DA, Dacic S, Giaccone G, Jenkins RB, et al. Molecular testing guideline for selection of lung cancer patients for EGFR and ALK tyrosine kinase inhibitors: guideline from the College of American Pathologists, International Association for the Study of Lung Cancer, and Association for Molecular Pathology. J Mol Diagn. 2013;15(4):415-453.

12. Liu X, Lu Y, Zhu G, Lei Y, Zheng L, Qin H, Tang C, et al. The diagnostic accuracy of pleural effusion and plasma samples versus tumour tissue for detection of EGFR mutation in patients with advanced non-small cell lung cancer: comparison of methodologies. J Clin Pathol. 2013;66(12):1065-1069.

13. Liu N, Sun RZ, Du J, Dong QZ, Fan CF, Li QC, Wang EH, et al. Comparison of epidermal growth factor receptor gene mutations identified using pleural effusion and primary tumor tissue samples in non-small cell lung cancer. Appl Immunohistochem Mol Morphol. 2018;26(4):e44e51.

14. Davies RS, Smith C, Edwards G, Butler R, Parry D, Lester JF. Impact of Cytological Sampling on EGFR Mutation Testing in Stage III-IV Lung Adenocarcinoma. Lung Cancer Int. 2017;2017:9614938.

15. Guan Y, Wang ZJ, Wang LQ, Hua DF, Liu J. Comparison of EGFR mutation rates in lung adenocarcinoma tissue and pleural effusion samples. Genet Mol Res. 2016;15(2):gmr.15027001.

16. Bray F, Ferlay J, Soerjomataram I, Siegel RL, Torre LA, Jemal A. Global cancer statistics 2018: GLOBOCAN estimates of incidence and mortality worldwide for 36 cancers in 185 countries. CA Cancer J Clin. 2018;68(6):394424.

17. Yotsukura M, Yasuda H, Shigenobu T, Kaseda K, Masai K, Hayashi Y, Hishida T, et al. Clinical and pathological characteristics of EGFR mutation in operable early-stage lung adenocarcinoma. Lung Cancer. 2017;109:45-51.

18. Rahman S, Kondo N, Yoneda K, Takuwa T, Hashimoto M, Orui H, Okumura Y, et al. Frequency of epidermal growth factor receptor mutations in Bangladeshi patients with adenocarcinoma of the lung. Int J Clin Oncol. 2014;19(1):45-49.

19. Zhou Y, Yang Y, Yang C, Chen Y, Yang C, Du Y, Zhao $\mathrm{G}$, et al. Epidermal growth factor receptor (EGFR) mutations in non-small cell lung cancer (NSCLC) of Yunnan in southwestern China. Oncotarget. 2017;8(9):1502315033.

20. Toh CK, Ahmad B, Soong R, Chuah KL, Tan SH, Hee SW, Leong SS, et al. Correlation between epidermal growth factor receptor mutations and expression of female hormone receptors in East-Asian lung adenocarcinomas. J Thorac Oncol. 2010;5(1):17-22.

21. Chang YL, Wu CT, Lin SC, Hsiao CF, Jou YS, Lee YC. Clonality and prognostic implications of p53 and epidermal growth factor receptor somatic aberrations in multiple primary lung cancers. Clin Cancer Res. 2007;13(1):5258.

22. Shi Y, Au JS, Thongprasert S, Srinivasan S, Tsai CM, Khoa MT, Heeroma K, et al. A prospective, molecular epidemiology study of EGFR mutations in Asian pa- tients with advanced non-small-cell lung cancer of adenocarcinoma histology (PIONEER). J Thorac Oncol. 2014;9(2):154-162.

23. Cooper WA, Yu B, Yip PY, Ng CC, Lum T, Farzin M, Trent RJ, et al. EGFR mutant-specific immunohistochemistry has high specificity and sensitivity for detecting targeted activating EGFR mutations in lung adenocarcinoma. J Clin Pathol. 2013;66(9):744-748.

24. Doval DC, Azam S, Batra U, Choudhury KD, Talwar V, Gupta SK, Mehta A. Epidermal growth factor receptor mutation in lung adenocarcinoma in India: A single center study. J Carcinog. 2013;12:12.

25. Maturu VN, Singh N, Bal A, Gupta N, Das A, Behera D. Relationship of epidermal growth factor receptor activating mutations with histologic subtyping according to International Association for the Study of Lung Cancer/ American Thoracic Society/European Respiratory Society 2011 adenocarcinoma classification and their impact on overall survival. Lung India. 2016;33(3):257-266.

26. Kasana BA, Dar WR, Aziz SA, Lone AR, Sofi NU, Dar IA, Latief M, et al. Epidermal growth factor receptor mutation in adenocarcinoma lung in a North Indian population: Prevalence and relation with different clinical variables. Indian J Med Paediatr Oncol. 2016;37(3):189-195.

27. Isaksson S, Bendahl PO, Salomonsson A, Jonsson M, Haglund M, Gaber A, Jirstrom K, et al. Detecting EGFR alterations in clinical specimens-pitfalls and necessities. Virchows Arch. 2013;463(6):755-764.

28. Frega S, Lorenzi M, Fassan M, Indraccolo S, Calabrese F, Favaretto A, Bonanno L, et al. Clinical features and treatment outcome of non-small cell lung cancer (NSCLC) patients with uncommon or complex epidermal growth factor receptor (EGFR) mutations. Oncotarget. 2017;8(20):32626-32638.

29. de Castro J, Tagliaferri P, de Lima VCC, Ng S, Thomas M, Arunachalam A, Cao X, et al. Systemic therapy treatment patterns in patients with advanced non-small cell lung cancer (NSCLC): PIvOTAL study. Eur J Cancer Care (Engl). 2017;26(6):e12734.

30. Arrieta O, Cardona AF, Federico Bramuglia G, Gallo A, Campos-Parra AD, Serrano S, Castro M, et al. Genotyping non-small cell lung cancer (NSCLC) in Latin America. J Thorac Oncol. 2011;6(11):1955-1959.

31. Gervas P, Ivanova A, Vasiliev N, Ananina O, Zharkova O, Rogovieva O, Verzhbitskaya N, et al. Frequency of EGFR mutations in non-small cell lung cancer patients: screening data from West Siberia. Asian Pac J Cancer Prev. 2015;16(2):689-692.

32. Leduc N, Atallah V, Agossou M, Vinh-Hung V, Orre M, Sargos P, Molinie V. Lung adenocarcinoma survival in EGFR-Mutated African-Caribbean patients: a multicenter study in the French West Indies. Target Oncol. 2017;12(5):689-693.

33. Sarosi V, Baliko Z, Smuk G, Laszlo T, Szabo M, Ruzsics I, Mezosi E. The Frequency of EGFR Mutation in Lung Adenocarcinoma and the Efficacy of Tyrosine Kinase Inhibitor Therapy in a Hungarian Cohort of Patients. Pathol Oncol Res. 2016;22(4):755-761.

34. De Greve J, Van Meerbeeck J, Vansteenkiste JF, Decos- 
ter L, Meert AP, Vuylsteke P, Focan C, et al. Prospective evaluation of first-line erlotinib in advanced Non-Small Cell Lung Cancer (NSCLC) carrying an activating EGFR mutation: a multicenter academic Phase II study in Caucasian patients (FIELT). PLoS One. 2016;11(3):e0147599.

35. Schmid-Bindert G, Wang Y, Jiang H, Sun H, Henzler T, Wang H, Pilz LR, et al. EBUS-TBNA provides highest RNA yield for multiple biomarker testing from routinely obtained small biopsies in non-small cell lung cancer patients - a comparative study of three different minimal invasive sampling methods. PLoS One. 2013;8(10):e77948.

36. Schmid K, Oehl N, Wrba F, Pirker R, Pirker C, Filipits M. EGFR/KRAS/BRAF mutations in primary lung adenocarcinomas and corresponding locoregional lymph node metastases. Clin Cancer Res. 2009;15(14):4554-4560.

37. Arrieta O, Cardona AF, Martin C, Mas-Lopez L, Corrales-Rodriguez L, Bramuglia G, Castillo-Fernandez O, et al. Updated frequency of EGFR and KRAS mutations in nonsmall-cell lung cancer in Latin America: The LatinAmerican Consortium for the Investigation of Lung Cancer (CLICaP). J Thorac Oncol. 2015;10(5):838-843.

38. Martin P, Shiau CJ, Pasic M, Tsao M, Kamel-Reid S, Lin $\mathrm{S}$, Tudor R, et al. Clinical impact of mutation fraction in epidermal growth factor receptor mutation positive NSCLC patients. Br J Cancer. 2016;114(6):616-622.

39. Lopez-Chavez A, Thomas A, Evbuomwan MO, Xi L, Chun G, Vidaurre T, Arrieta O, et al. EGFR Mutations in Latinos From the United States and Latin America. J Glob Oncol. 2016;2(5):259-267.

40. Sriram KB, Tan ME, Savarimuthu SM, Wright CM, Relan $\mathrm{V}$, Stockwell RE, Clarke BE, et al. Screening for activating EGFR mutations in surgically resected nonsmall cell lung cancer. Eur Respir J. 2011;38(4):903-910.
41. Hata A, Yoshioka H, Fujita S, Kunimasa K, Kaji R, Imai $\mathrm{Y}$, Tomii K, et al. Complex mutations in the epidermal growth factor receptor gene in non-small cell lung cancer. J Thorac Oncol. 2010;5(10):1524-1528.

42. Kauffmann-Guerrero D, Huber RM, Reu S, Tufman A, Mertsch P, Syunyaeva Z, Jung A, et al. NSCLC patients harbouring rare or complex EGFR mutations are more often smokers and might not benefit from first-line tyrosine kinase inhibitor therapy. Respiration. 2018;95(3):169176.

43. Hsu F, De Caluwe A, Anderson D, Nichol A, Toriumi T, Ho C. Patterns of spread and prognostic implications of lung cancer metastasis in an era of driver mutations. Curr Oncol. 2017;24(4):228-233.

44. Rekhtman N, Paik PK, Arcila ME, Tafe LJ, Oxnard GR, Moreira AL, Travis WD, et al. Clarifying the spectrum of driver oncogene mutations in biomarker-verified squamous carcinoma of lung: lack of EGFR/KRAS and presence of PIK3CA/AKT1 mutations. Clin Cancer Res. 2012;18(4):1167-1176.

45. Ragazzi M, Tamagnini I, Bisagni A, Cavazza A, Pagano M, Baldi L, Boni C, et al. Diamond: immunohistochemistry versus sequencing in EGFR analysis of lung adenocarcinomas. J Clin Pathol. 2016;69(5):440-447.

46. Zhang R, Li Y, Nie X, Dong X, Wu G. Prognostic implications of immunohistochemistry markers for EGFR-TKI therapy in Chinese patients with advanced lung adenocarcinoma harboring EGFR mutations. Onco Targets Ther. 2016;9:355-366.

47. Kitamura A, Hosoda W, Sasaki E, Mitsudomi T, Yatabe Y. Immunohistochemical detection of EGFR mutation using mutation-specific antibodies in lung cancer. Clin Cancer Res. 2010;16(13):3349-3355. 